\title{
Três Hipóteses sobre a Natureza das Tarefas de Nomeação Seriada Rápida
}

\author{
Cláudia Nascimento Guaraldo Justi ${ }^{1}$ \\ Antonio Roazzi \\ Francis Ricardo dos Reis Justi \\ Flávia Guimarães Henriques \\ Mariana Figueira Lopes Cançado \\ Universidade Federal de Juiz de Fora
}

\begin{abstract}
RESUMO - O presente estudo investigou a natureza das tarefas de nomeação seriada rápida, considerando três hipóteses sobre o que elas representariam: (1) medidas da recuperação de códigos fonológicos da memória de longo prazo; (2) medidas da velocidade de processamento geral; e (3) medidas de processos relacionados ao reconhecimento de padrões visuais. Participaram da pesquisa 174 crianças brasileiras cursando o $5^{\circ}$ ano do Ensino Fundamental em escolas particulares. Análises fatoriais confirmatórias indicaram que nenhuma das três hipóteses se mostrou consistente com os dados. Porém, em um modelo de regressão estrutural construído para avaliar a contribuição desses fatores para a nomeação seriada rápida, o único que contribuiu significativamente foi o fator 'velocidade de processamento geral'.
\end{abstract}

Palavras-chave: nomeação seriada rápida, processamento fonológico, velocidade de processamento geral, processamento visual, leitura

\section{Three Hypotheses on the Nature of Rapid Automatized Naming Tasks}

\begin{abstract}
The present study aimed to investigate the nature of rapid automatized naming tasks, considering three hypotheses about what they represent: (1) measures of retrieval of phonological codes from a long-term store; (2) measures of general processing speed; and (3) measures of visual pattern recognition processes. The participants were 174 children from the 5 th grade of Brazilian private schools. Confirmatory factor analyses indicated that none of the hypotheses were consistent with the data. However, in a structural regression model the factor 'general processing speed' was the only one that contributed significantly to rapid automatized naming.
\end{abstract}

Keywords: rapid automatized naming, phonological processing, general processing speed, visual processing, reading

A leitura está entre as aquisições mais significativas da mente humana, assim como está entre as habilidades mais criticamente requeridas nas sociedades modernas. Perspectivas atuais sugerem que o aprendizado da leitura é um processo complexo e multifacetado, formado sobre uma variedade de habilidades que emergem e se desenvolvem cedo na infância (Grigorenko \& Naples, 2008). Considerando-se o aspecto cognitivo da leitura, uma das variáveis que vem sendo, sistematicamente, apontada como sendo preditiva do desenvolvimento da leitura é a nomeação seriada rápida (Barth, Catts, \& Anthony, 2009; Cronin, 2011; Cutting \& Denckla, 2001; Georgiou, Parrila, Cui, \& Papadopoulos, 2013; Katzir, Kim, Wolf, Morris \& Lovett, 2008; Kirby, Pfeiffer, \& Pariila, 2003; Meyer, Wood, Hart, \& Felton, 1998; Schatschneider, Carlson, Francis, Foorman, \& Fletcher, 2004; Wagner \& Torgesen, 1987; Wolf \& Bowers, 1999).

A primeira demonstração de que crianças com dificuldade de leitura podem apresentar dificuldade na nomeação seriada rápida foi realizada por Denckla e Rudel (1976). Essas pesquisadoras elaboraram a tarefa denominada

1 Endereço para correspondência: Instituto de Ciências Humanas, Universidade Federal de Juiz de Fora, Campus Universitário, Juiz de Fora, MG, Brasil, CEP: 36036-330. E-mail: claudia.ngjusti@gmail.com
Rapid Automatized Naming (Nomeação Seriada Rápida) ${ }^{1}$ que acabou se tornando uma medida padrão para avaliar a nomeação seriada rápida. Em uma tarefa de nomeação seriada rápida, o participante nomeia, tão rápida e corretamente quanto possível, um conjunto de 50 estímulos visuais, todos de alta frequência de ocorrência, dispostos em série em um cartão. Os estímulos visuais a serem nomeados podem ser: quadrados coloridos - na tarefa de nomeação seriada rápida de cores; números - na tarefa de nomeação seriada rápida de números; objetos desenhados - na tarefa de nomeação seriada rápida de objetos; e, letras - na tarefa de nomeação seriada rápida de letras. Por exemplo: a tarefa de nomeação seriada rápida de letras, desenvolvida por Denckla e Rudel, consiste na apresentação de um cartão contendo cinco letras (a, d, o, $\mathrm{s}, \mathrm{p})$. Cada letra aparece 10 vezes, em ordem aleatória, em uma matriz composta por cinco fileiras e 10 colunas.

1 Optou-se, neste estudo, por traduzir 'Rapid Automatized Naming' por 'Nomeação Seriada Rápida' para enfatizar o aspecto serial dessa tarefa. Há evidências de que esse aspecto é crucial para a relação encontrada entre o desempenho nessa tarefa e o desempenho em tarefas que avaliam a leitura (Logan \& Schatschneider, 2014; Logan, Schatschneider \& Wagner, 2011). É importante mencionar que, de uma forma geral, pesquisadores utilizam o termo 'rapid automatized naming' para se referirem tanto à tarefa, quanto ao construto. O mesmo procedimento foi adotado no presente trabalho com o termo 'nomeação seriada rápida'. 
Wolf, Bally e Morris (1986) mostraram que todas as quatro tarefas de nomeação seriada rápida (cores, números, objetos e letras) aplicadas em crianças na pré-escola, predisseram as variações nas habilidades de leitura (precisão, fluência e compreensão) quando as mesmas crianças estavam na $2^{\mathrm{a}}$ série. Uma vez que as tarefas de nomeação seriada rápida podem ser realizadas mesmo por crianças que ainda não iniciaram o aprendizado formal da leitura, os achados relativos ao poder da tarefa em predizer o desenvolvimento da leitura têm tornado a nomeação seriada rápida uma das tarefas mais importantes para identificar crianças em risco de apresentar dificuldade de leitura (Bishop \& League, 2006; Puolakanaho et al., 2007).

Atualmente, há um número substancial de estudos que evidenciam que o desempenho em tarefas de nomeação seriada rápida é preditivo do desempenho em tarefas de leitura, mesmo entre crianças aprendendo a ler em diversos sistemas alfabéticos, como o turco (Babayigit \& Stainthorp, 2010), o alemão (Landerl \& Wimmer, 2008; Wimmer, Mayringer, \& Landerl, 2000), o norueguês (Lervåg, Bråten, \& Hulme, 2009), o finlandês (Lepola, Poskiparta, Laakkonen, \& Niemi, 2005; Torppa et al., 2013), o holandês (de Jong \& van der Leij, 2003; Verhagen, Aarnoutse, \& van Leeuwe, 2008), o espanhol (Escribano, 2007; López-Escribano, \& Katzir, 2008), o italiano (Brizzolara et al., 2006; Di Filippo et al., 2006), o inglês (Kirby et al., 2003; Powell, Stainthorp, Stuart, Garwood \& Quinlan, 2007), o francês (Plaza, 2003; Plaza \& Cohen, 2004) e o português brasileiro (Justi \& Roazzi, 2012). No entanto, a natureza dessa relação ainda não é clara.

Uma das teorias mais conhecidas no âmbito dos estudos científicos sobre a leitura e a escrita é a teoria do processamento fonológico (Bradley \& Bryant, 1978; Brady \& Shankweiler, 1991; Wagner \& Torgesen, 1987). De acordo com essa teoria, uma dificuldade no uso da informação fonológica seria a principal causa de dificuldade na aquisição da leitura e da escrita. Wagner e Torgesen (1987) e Wagner, Torgesen, Laughon, Simmons e Rashotte (1993) propõem que a consciência fonológica (habilidade de perceber e manipular os sons da fala), a codificação fonológica na memória de trabalho e a recuperação de códigos fonológicos da memória de longo prazo são três componentes do processamento fonológico, sendo as tarefas de nomeação seriada rápida medidas desse último componente. Assim sendo, as tarefas de nomeação seriada rápida avaliariam a recuperação de códigos fonológicos da memória de longo prazo e, por esse motivo, relacionar-se-iam com o desenvolvimento da leitura. Para esses pesquisadores: “.... eficiência com que as crianças são capazes de recuperar códigos fonológicos associados com fonemas individuais, palavras segmentadas ou palavras inteiras influencia o grau em que a informação fonológica é aproveitada na decodificação" (Wagner et al., 1993, p. 84).

Para avaliar se a nomeação seriada rápida é uma medida do processamento fonológico, pesquisadores têm realizado análises de regressão em que uma medida de nomeação seriada rápida é incluída na equação regressiva após as medidas de consciência fonológica ou de memória de trabalho fonológica (representantes de variações no processamento fonológico). De uma forma geral, podese dizer que os resultados desses estudos evidenciam que a nomeação seriada rápida permanece contribuindo para a leitura mesmo após o controle de variações no processamento fonológico, conforme indexadas pelas medidas de consciência fonológica ou de memória de trabalho fonológica (Babayigit \& Stainthorp, 2010; Cutting \& Denckla, 2001; de Jong \& van der Leij, 2003; Justi \& Roazzi, 2012; Kirby et al., 2003; Landerl \& Wimmer, 2008; Lepola et al., 2005; Lervåg et al., 2009; López-Escribano, \& Katzir, 2008; Meyer et al., 1998; Plaza, 2003; Plaza \& Cohen, 2004; Schatschneider, Carlson, Francis, Foorman, \& Fletcher, 2004; Wimmer et al., 2000). Esse tipo de resultado sugere que a nomeação seriada rápida avalia processos não fonológicos e que esses processos também estariam relacionados com a habilidade de leitura. No entanto, conforme pontuado por Logan et al. (2011), esse resultado também é compatível com a proposta de Wagner e Torgesen (1987), já que nessa as tarefas de consciência fonológica, de memória de trabalho fonológica e de nomeação seriada rápida seriam medidas de processos fonológicos distintos (sendo eles, respectivamente, a habilidade de perceber e manipular os sons da fala, a codificação fonológica na memória de trabalho e a recuperação de códigos fonológicos da memória de longo prazo). Dessa forma, possivelmente, uma avaliação mais precisa da proposta de que a nomeação seriada rápida seria uma medida da recuperação de códigos fonológicos da memória de longo prazo implicaria na inclusão de outra medida de recuperação de códigos fonológicos da memória de longo prazo, a fim de se analisar se a nomeação seriada rápida continuaria contribuindo para a leitura, após o controle dessa variável.

Uma explicação alternativa para a relação existente entre a nomeação seriada rápida e a leitura foi proposta por Kail e Hall (1994). Eles sugeriram que o desempenho nas tarefas de nomeação seriada rápida seria um índice da velocidade de processamento geral, uma vez que o tempo que as crianças levam para realizar essas tarefas diminui à medida que elas vão crescendo. Dessa forma, o aumento na velocidade das crianças em nomear os estímulos visuais presentes nas tarefas de nomeação seriada rápida seria apenas uma manifestação de um aumento na velocidade de processamento geral causado pelo desenvolvimento e crescimento relacionados à idade. Essa interpretação fundamenta-se no fato de que, com o desenvolvimento, as crianças processam, de uma forma geral, informações mais rapidamente. Por exemplo, enquanto crianças de quatro anos processam informações três vezes mais lentamente do que os adultos, crianças de oito anos processam informações duas vezes mais lentamente do que os adultos. Kail e Hall argumentam, também, que as variações na velocidade de processamento geral é que explicariam a relação existente entre o desempenho em tarefas de nomeação seriada rápida e o desempenho em tarefas de leitura.

Há evidências de que o desempenho em tarefas de nomeação seriada rápida correlaciona-se significativamente com o desempenho em tarefas de velocidade de processamento geral (Bowey, McGuigan, \& Ruschena, 2005; Kail \& Hall; 1994; Kail, Hall \& Caskey, 1999; Powell et al., 2007). Além disso, o estudo de Catts, Gillispie, Leonard, Kail e Miller (2002), realizado com 279 crianças que foram acompanhadas da $2 \mathrm{a}$ à $4 \mathrm{a}$ série, apresenta evidências de que seriam, exatamente, as variações compartilhadas entre a nomeação seriada rápida e a velocidade de processamento 
geral que explicariam a relação entre a nomeação seriada rápida e a leitura. De uma forma geral, os resultados desse estudo evidenciaram que, ao incluir a medida de nomeação seriada rápida após a medida de velocidade de processamento geral em uma equação regressiva, a nomeação seriada rápida deixou de contribuir para a leitura, mostrando que a velocidade de processamento geral explicaria completamente a relação existente entre a nomeação seriada rápida e a leitura. No entanto, há inconsistência nos resultados dos estudos que investigaram a proposta de Kail e Hall (1994). O estudo de Powell et al. (2007), por exemplo, sugere o oposto do que foi obtido no estudo de Catts et al. Em seu estudo, Powell et al. realizaram análises de regressão e encontraram que mesmo quando a velocidade de processamento geral entrou primeiro na equação regressiva, a nomeação seriada rápida ainda explicou, significativamente, $17 \%$ das variações na habilidade de leitura. Tendo em vista os achados conflitantes, torna-se importante a realização de mais estudos que investiguem a hipótese de Kail e Hall.

Por fim, outra hipótese acerca da relação entre a nomeação seriada rápida e a leitura tem sido proposta por Bowers (2001). A pesquisadora sugere que a nomeação seriada rápida poderia indexar processos que são refletidos em medidas de linha de base de identificação de conjuntos de letras com baixo padrão de estrutura ortográfica (conjuntos pouco usuais de letras). A pesquisadora se inspirou no resultado do estudo de Bowers, Sunseth e Golden (1999) para elaborar essa hipótese. Nesse estudo, crianças com lenta nomeação seriada, quando comparadas a crianças que tinham dificuldade na consciência fonológica, mas não na nomeação seriada rápida, apresentaram desempenho pior quando tiveram que relatar se uma letra estava contida em um conjunto de letras apresentados anteriormente, quando esse conjunto de letras provinha de uma combinação pouco usual. Dessa forma, a hipótese de Bowers sugere que um lento desempenho em tarefas de nomeação seriada rápida refletiria processos resultantes de um processamento incompleto de conjuntos de letras com baixo padrão de estrutura ortográfica. Assim sendo, essa hipótese sugere que a nomeação seriada rápida afeta a habilidade de reconhecimento visual de palavras porque a nomeação seriada rápida indexaria a velocidade com que as características visuais dos estímulos são mapeadas nas letras que os compõem em um processo do tipo botton up (e.g., a velocidade com que o conjunto de características '/l' é mapeado como correspondendo à letra 'A'). Apesar dessa hipótese ter sido motivada por um dado empírico (a associação de um desempenho ruim na tarefa de nomeação seriada rápida com o aumento no relato incorreto de letras presentes em conjuntos com baixa estrutura ortográfica), ela ainda não parece ter sido avaliada em estudos independentes.

Em suma, enquanto a relação entre o desempenho em tarefas de nomeação seriada rápida e a habilidade de leitura tem sido empiricamente demonstrada, ainda não está claro quais processos estariam mediando essa relação, uma vez que ainda permanece obscura a natureza das tarefas de nomeação seriada rápida. Compreender melhor quais processos cognitivos subjazem o desempenho em tarefas de nomeação seriada rápida é um passo importante para a realização de um diagnóstico adequado de dificuldades de leitura, bem como é um passo indispensável para a elaboração de um programa de intervenção que tenha como objetivo estimular esses processos, visando o desenvolvimento da leitura. Assim sendo, o presente estudo investigou, por meio da modelagem de equação estrutural, a consistência com os dados de três hipóteses explicativas sobre a natureza das tarefas de nomeação seriada rápida, a saber: (1) a hipótese de que as tarefas de nomeação seriada rápida seriam medidas da recuperação de códigos fonológicos na memória de longo prazo (Wagner \& Torgesen, 1987); (2) a hipótese de que as tarefas de nomeação seriada rápida seriam medidas da velocidade de processamento geral (Kail \& Hall, 1994); e (3) a hipótese de que a nomeação seriada rápida indexaria processos perceptuais responsáveis pelo mapeamento das características visuais de um estímulo (Bowers, 2001).

É importante ressaltar que este é o primeiro estudo realizado com falantes do português brasileiro com o objetivo de investigar as hipóteses de Kail e Hall (1994) e Bowers (2001), sendo importante destacar, também, a carência de pesquisas sobre essas duas hipóteses na literatura internacional. Além disso, o presente estudo utilizou medidas de recuperação de códigos fonológicos da memória de longo prazo para investigar a hipótese de Wagner e Torgesen (1987). A inclusão desse tipo de medida é importante, tendo em vista que esses autores argumentam que a nomeação seriada rápida seria uma medida da recuperação de códigos fonológicos da memória de longo prazo, sendo esse um processo fonológico diferente dos processos avaliados pelas tarefas de consciência fonológica e de memória de trabalho fonológica (tarefas tradicionalmente incluídas nos estudos que avaliaram a hipótese desses pesquisadores). Uma vantagem adicional do presente estudo é o fato dessas três hipóteses terem sido avaliadas em uma mesma amostra.

\section{Método}

\section{Participantes}

Participaram do presente estudo 174 crianças do $5^{\circ}$ ano do Ensino Fundamental de escolas particulares de uma cidade de médio porte (aproximadamente 550.000 habitantes) do estado de Minas Gerais. A idade média das crianças era de 10 anos e 6 meses (desvio padrão de 6,27 meses). A participação das crianças na pesquisa foi condicionada à assinatura, pelos responsáveis, do Termo de Consentimento Livre e Esclarecido. Essa pesquisa foi aprovada pelo Comitê de Ética em Pesquisa da instituição à qual a primeira autora está vinculada (processo no 384.929).

\section{Instrumentos}

Medida de recuperação de códigos fonológicos da memória de longo prazo. Foi utilizado o Teste de Fluência de Aliteração (baseado em Cardoso-Martins, Haase, \& Wood, 1998). Esse teste requer que a criança diga, em um período de $30 \mathrm{~s}$, a maior quantidade possível de palavras que começam com um som específico determinado pelo examinador. No presente estudo os sons escolhidos foram o som /s/ e o som 
/x/. O escore para cada som escolhido consistiu no número de palavras produzidas corretamente no período de $30 \mathrm{~s}$. A aplicação do teste contou com uma sessão prévia de treinamento na qual as crianças deviam dizer, em $30 \mathrm{~s}$, o maior número possível de palavras que começassem com o som /R/. Caso as crianças tivessem alguma dúvida durante o treino, o pesquisador fornecia exemplos de palavras com o som /R/ no início. A aplicação da sessão principal do teste ocorreu logo após a sessão de treinamento.

Medidas de velocidade de processamento geral. Foram utilizados dois subtestes: Código e Procurar Símbolos, ambos do WISC III (Wechsler, 2002). No Subteste Código é esperado que cada criança copie símbolos simples que são pareados com números. A criança tem que desenhar cada símbolo embaixo do seu número correspondente de acordo com um modelo. Por exemplo, abaixo do número ' 3 ' a criança sempre deve desenhar o símbolo ' + '. O escore da criança é determinado pelo número de símbolos corretamente desenhados em 120 s. No Subteste Procurar Símbolos, para cada item, a criança examina, visualmente, dois grupos de símbolos: um grupo padrão e um grupo de busca. $\mathrm{O}$ grupo padrão contém dois símbolos como modelo e o grupo de busca tem cinco símbolos. A criança indica, marcando no quadro apropriado, se uma das figuras do modelo aparece no grupo de busca. $\mathrm{O}$ escore consiste no número de itens corretamente marcados em um limite de tempo de $120 \mathrm{~s}$. Ambos os subtestes contêm itens de exemplo no início que são utilizados pelo pesquisador para explicar e demonstrar a execução da tarefa. A aplicação dos dois subtestes obedeceu, integralmente, às instruções contidas no manual do WISC III (Wechsler, 2002).

Medidas de nomeação seriada rápida. Foram utilizadas duas tarefas: Nomeação Seriada Rápida de Letras e Nomeação Seriada Rápida de Números (adaptadas por Justi \& Roazzi, 2012, de Denckla \& Rudel, 1976). Para a realização da Tarefa de Nomeação Seriada Rápida de Letras, foi solicitado que a criança nomeasse, da forma mais rápida e correta quanto possível, as letras 'a', 'r', 's', 'o', 'm', dispostas serialmente em um cartão. Cada letra aparecia 10 vezes, em ordem aleatória, em cinco fileiras horizontais. Para a realização da Tarefa de Nomeação Seriada Rápida de Números, foi solicitado que a criança nomeasse, da forma mais rápida e correta quanto possível, os números ' 8 ', '4', '7', '5', '9', dispostos serialmente em um cartão. Cada número aparecia 10 vezes, em ordem aleatória, em cinco fileiras horizontais. Em ambas as tarefas, o escore consistiu no tempo gasto pelas crianças para nomear todos os estímulos presentes no cartão. Para o registro do tempo foi utilizado um cronômetro digital com precisão de centésimos de segundo. Antes de cada tarefa, era apresentado um cartão de treino com os cinco estímulos (letras ou números) que estariam presentes na tarefa, cabendo à criança dizer o nome de cada um desses estímulos. Durante o treinamento, se a criança não soubesse o nome de um dos estímulos, o pesquisador lhe dizia a resposta correta e a tarefa só era aplicada quando os cinco estímulos eram nomeados corretamente pela criança.

Medidas de processamento visual. Foram utilizadas duas tarefas: Tarefa de Julgamento da Semelhança de dois Conjuntos de Letras Não Pronunciáveis - TJS (baseada em Seymour, 1986) e Tarefa de Reconhecimento de Letras - TRL (baseada em Bowers et al., 1999). Na TJS são apresentadas, simultaneamente, no centro da tela de um computador, duas sequências de cinco consoantes escritas em letra maiúscula, uma ao lado da outra, por um breve período de tempo. A criança deve pressionar um botão verde, se as duas sequências são idênticas e um botão vermelho, se são diferentes. Na TRL é apresentado, no centro da tela do computador, um conjunto de cinco letras por um breve período de tempo (250ms). Após o desaparecimento do conjunto de letras, aparece uma letra. A criança deve pressionar um botão verde, caso essa letra estivesse presente no estímulo anterior, ou um botão vermelho, caso essa letra não estivesse presente no estímulo anterior. Para assegurar que os conjuntos de letras tivessem um baixo padrão de estrutura ortográfica, foram utilizadas sequências aleatórias de consoantes. Para ambas as tarefas, o software $D M D X$ (Forster \& Forster, 2003) foi utilizado para a apresentação dos estímulos e para o cômputo da porcentagem de erros de cada participante. A aplicação de ambas as tarefas (TJS e TRL) contou com uma sessão prévia de treinamento. Nessa sessão, após cada resposta, a criança recebia um feedback, na tela do computador, que indicava se a sua resposta estava correta ou errada. Além disso, o treinamento também servia para os participantes poderem dirimir quaisquer dúvidas que tivessem, antes de iniciarem a sessão principal que ocorria, imediatamente, após a sessão de treino.

\section{Procedimento}

As tarefas foram aplicadas em uma sala reservada, disponibilizada pela própria escola, após a autorização dos responsáveis pelas crianças. As crianças foram testadas individualmente, em dois dias diferentes. No primeiro dia foram aplicados os testes de Fluência de Aliteração com o som /s/ e com o som /x/, os subtestes Código e Procurar Símbolos, e as tarefas de Nomeação Seriada Rápida de Números e de Letras. No segundo dia foram aplicadas a TJS e a TRL. A duração estimada do período de testagem, em cada dia, foi de, aproximadamente, 20 minutos. Os testes foram aplicados por pesquisadores treinados e o intervalo de tempo entre os dois dias de testagem foi de, aproximadamente, uma semana.

\section{Resultados}

No que se refere à distribuição das variáveis incluídas no estudo, excetuando as variáveis 'nomeação seriada rápida de números', 'nomeação seriada rápida de letras' e 'código', as demais variáveis apresentaram uma distribuição normal (assimetria e curtose com valores entre $1 \mathrm{e}-1$ ). Assim sendo, as variáveis que não apresentaram uma distribuição normal foram submetidas a uma transformação logarítmica $(\lg 10)$, uma vez que apresentaram uma distribuição positivamente assimétrica. Esse procedimento foi suficiente para garantir a distribuição normal de todas as variáveis do estudo e, além disso, a normalidade multivariada dos dados (o valor do coeficiente de Mardia ficou entre 1,96 e -1,96; Garson, 2012). 
Com base nas propostas de Wagner e Torgesen (1987), Kail e Hall (1994) e Bowers (2001), três modelos de mensuração foram elaborados e avaliados por meio de análises fatoriais confirmatórias. $\mathrm{O}$ método utilizado para o cálculo das estimativas foi o de Máxima Verossimilhança (do inglês Maximum Likelihood - ML).

O primeiro modelo avaliado representou a proposta de Wagner e Torgesen (1987), segundo a qual a nomeação seriada rápida é uma medida da recuperação de códigos fonológicos da memória de longo prazo. Assim sendo, nesse modelo, um fator geral denominado 'Processamento Fonológico' foi indexado pelas medidas 'Nomeação Seriada Rápida de Números', 'Nomeação Seriada Rápida de Letras', 'Fluência de Aliteração com o som /s/' e 'Fluência de Aliteração com o som /x/'. No segundo modelo, o fator 'Velocidade de Processamento Geral' foi indexado pelas medidas de 'Nomeação Seriada Rápida de Números', 'Nomeação Seriada Rápida de Letras', 'Código' e 'Procurar Símbolos', representando, dessa forma, a proposta de Kail e Hall (1994). Por fim, o terceiro modelo representou a proposta de Bowers (2001) de que a nomeação seriada rápida é uma medida do processamento visual de baixo nível, ao definir o fator 'Processamento Visual' como sendo indexado pelas medidas de 'Nomeação Seriada Rápida de Números', 'Nomeação Seriada Rápida de Letras', 'Julgamento de Semelhança' e 'Reconhecimento de Letras'.

Para a avaliação do ajuste dos modelos teóricos aos dados é comum os pesquisadores relatarem o resultado do teste qui-quadrado $\left(\chi^{2}\right)$ e o $p$ associado a ele. $\mathrm{O} \chi^{2}$ é um índice da significância da diferença entre a matriz de dados observada e a matriz estimada (modelo teórico proposto). Um bom modelo apresenta um $p$ associado ao valor de $\chi^{2}$ não significativo, indicando que o modelo teórico proposto não difere significativamente dos dados. Outro índice que também é utilizado é o Índice de Qualidade do Ajuste (do inglês Goodness of Fit Index - GFI). Este índice é uma estimativa da proporção de variância explicada, com valores de GFI $>0,90$ indicando um bom ajuste do modelo aos dados e valores de $\mathrm{GFI}<0,90$ indicando um ajuste pobre. De acordo com Kline (2005), o Índice de Ajuste Comparativo (do inglês Comparative Fit Index - CFI) e a Raiz Quadrada Média do Erro de Aproximação (do inglês Root Mean Square Error of Approximation - RMSEA) são os melhores índices do ajuste de um modelo aos dados por serem menos sensíveis ao tamanho da amostra. O CFI compara o modelo em teste com um modelo em que não há nenhuma covariação entre as variáveis. Os valores do CFI variam entre 0 e 1 , sendo os valores acima de 0,95 indicativos de que o modelo proposto é um bom modelo. Por outro lado, o RMSEA é uma estimativa da quantidade de erros de aproximação do modelo dividido por seus graus de liberdade. Os valores do RMSEA variam entre 0 e 1 , sendo os valores abaixo de 0,08 indicativos de um bom modelo. Assim sendo, para avaliar o ajuste dos modelos teóricos aos dados, esses quatro índices foram adotados no presente estudo. A Tabela 1 apresenta esses índices para os três modelos testados.

Os resultados da avaliação do ajuste desses três modelos revelaram que nenhum deles foi consistente com os dados. $\mathrm{O}$ modelo 1 (representante da proposta de Wagner \& Torgesen, 1987) apresentou um valor de $\mathrm{p}$ associado ao qui-quadrado significativo $\left[\chi^{2}(2, \mathrm{n}=174)=7,64, p=0,022\right]$, indicando que o modelo proposto e os dados observados foram significativamente diferentes. Apesar dos índices adequados de GFI $(0,979)$ e CFI $(0,957)$, o modelo apresentou um RMSEA igual a 0,128 , outro indício de um modelo ruim. $\mathrm{O}$ modelo 2 (representante da proposta de Kail \& Hall, 1994) também apresentou um valor de $\mathrm{p}$ associado ao qui-quadrado significativo $\left[\chi^{2}(2, \mathrm{n}=174)=76,735, p=0,000\right]$, indicando, também, que o modelo proposto e os dados observados foram significativamente diferentes, o que foi corroborado pelos demais índices. O modelo 3 (representante da proposta de Bowers, 2001) - assim como os anteriores, apresentou um valor de $\mathrm{p}$ associado ao qui-quadrado significativo $\left[\chi^{2}(2, \mathrm{n}=\right.$ 174) $=18,877, p=0,000]$, indicando, também, que o modelo proposto e os dados observados foram significativamente diferentes. Os demais índices, com exceção do GFI, também indicam que não se trata de um bom modelo.

Tendo em vista esses resultados, um modelo de regressão estrutural foi desenvolvido para verificar o quanto da variação na nomeação seriada rápida se deve a variações no processamento fonológico, na velocidade de processamento geral e no processamento visual. Esse modelo (Modelo 4) representa a ideia de que mesmo que a nomeação seriada rápida não possa ser vista como uma medida específica de qualquer um desses construtos, variações na nomeação seriada rápida podem refletir variações no processamento fonológico, na velocidade de processamento geral e no processamento visual, em maior ou menor grau. A Figura 1 apresenta a representação gráfica do Modelo 4.

Os resultados da avaliação do Modelo 4 revelaram que o mesmo foi consistente com os dados. O modelo apresentou um valor de $\mathrm{p}$ associado ao qui-quadrado não significativo [Modelo4: $\left.\chi^{2}(2, n=174)=20,372, p=0,119\right]$ e os demais índices de ajuste indicaram que se trata de

Tabela 1. Índices de Ajuste dos Modelos aos Dados

\begin{tabular}{ccccccc}
\hline Modelos & $\boldsymbol{X}^{\mathbf{2}}$ & $\mathbf{p}$ & GI & GFI & CFI & RMSEA \\
\hline 1 & 7,64 & 0,022 & 2 & 0,979 & 0,957 & 0,128 \\
2 & 76,735 & 0,001 & 2 & 0,836 & 0,731 & 0,465 \\
3 & 18,877 & 0,001 & 2 & 0,951 & 0,874 & 0,221 \\
\hline
\end{tabular}

Nota . Modelo 1 = modelo representando a proposta de Wagner e Torgesen (1987); Modelo 2 = modelo representando a proposta de Kail e Hall (1994); Modelo 3 = modelo representando a proposta de Bowers (1999); GFI (Goodness of Fit Index) = Índice de Qualidade do Ajuste; CFI (Comparative Fit Index) = Índice de Ajuste Comparativo; RMSEA (Root Mean Square Error of Approximation $)=$ Raiz Quadrada Média do Erro de Aproximação. 


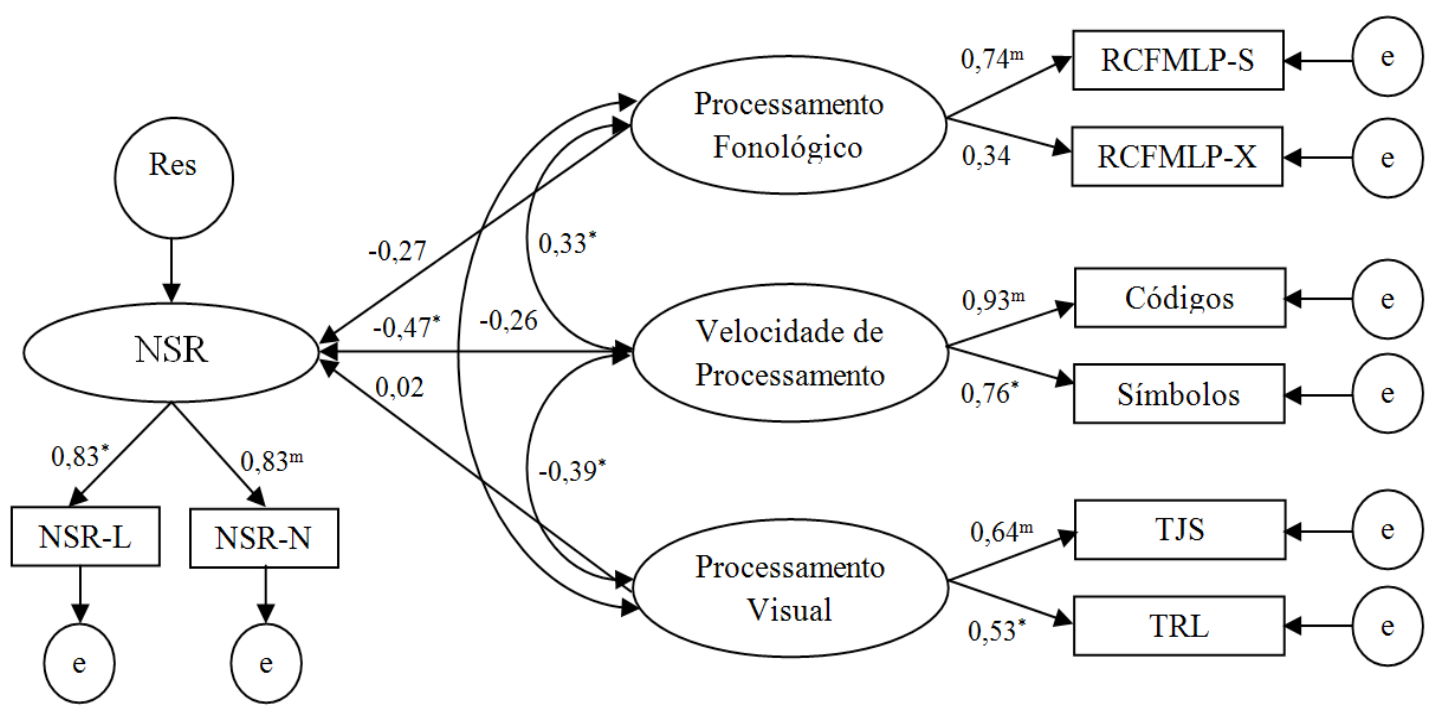

Figura 1. Diagrama do modelo de regressão estrutural. NSR = nomeação seriada rápida; NSR-L = nomeação seriada rápida de letras; NSR-N = nomeação seriada rápida de números; RCFMLP-S = recuperação de códigos fonológicos da memória de longo prazo som /s/; RCFMLP-X = recuperação de códigos fonológicos da memória de longo prazo som $/ \mathrm{x} /$; TJS = tarefa de julgamento de semelhança; $\mathrm{TRL}=$ tarefa de reconhecimento de letras; Res $=$ resíduo; $\mathrm{e}=$ erro de medida; $(\mathrm{m})=$ métrica; ${ }^{*} p<0,05$.

um bom modelo $(\mathrm{GFI}=0,971 ; \mathrm{CFI}=0,981$; $\mathrm{RMSEA}=$ 0,051). A análise desse modelo possibilitou evidenciar que, dentre as três variáveis latentes (processamento fonológico, velocidade de processamento geral e processamento visual), a variável 'velocidade de processamento geral' foi a única que explicou, de forma significante, as variações na variável endógena 'nomeação seriada rápida' $(\beta=-0,47)$. Além disso, a variável 'velocidade de processamento' foi a única que se correlacionou de forma estatisticamente significante com as demais, apresentando uma correlação de $-0,387$ com o 'processamento visual' e uma correlação de 0,322 com a 'recuperação de códigos fonológicos da memória de longo prazo - RCFMLP'.

\section{Discussão}

Tendo em vista os vários estudos que evidenciaram uma correlação significativa entre a nomeação seriada rápida e a leitura, bem como a falta de clareza sobre a natureza das tarefas de nomeação seriada rápida, o presente estudo teve como objetivo verificar a consistência com os dados de três hipóteses teóricas tecidas a esse respeito: a hipótese de Wagner e Torgesen (1987), a hipótese de Kail e Hall (1994) e a hipótese de Bowers (2001). Ao analisar diretamente cada uma dessas três hipóteses por meio da técnica de análise fatorial confirmatória, os índices dos modelos testados revelaram que nenhuma dessas hipóteses é satisfatoriamente consistente com os dados. Esse é um resultado importante porque indica que a nomeação seriada rápida não pode ser explicada, exclusivamente, por qualquer um dos processos postulados, a saber: a recuperação de códigos fonológicos da memória de longo prazo (Wagner \& Torgesen, 1987), a velocidade de processamento geral (Kail \& Hall, 1994) e o processamento visual de baixo nível (Bowers, 2001).

No que diz respeito à hipótese de Wagner \& Torgesen (1987) de que a nomeação seriada rápida seria uma medida da recuperação de códigos fonológicos da memória de longo prazo, pode-se dizer que os resultados do presente estudo estão de acordo com os de outros estudos recentes que têm demonstrado que a natureza das tarefas de nomeação seriada rápida não é prioritariamente fonológica (Justi, Roazzi \& Justi, 2014; Logan et al., 2011; Nelson, Lindstrom, Lindstrom, \& Denis, 2012). Pode-se, inclusive, dizer que o presente estudo complementa os estudos de Justi et al. (2014) e de Nelson et al. (2012) porque esses estudos utilizaram apenas medidas de consciência fonológica e de memória de trabalho fonológica, não utilizando, dessa forma, medidas representativas da recuperação de códigos fonológicos da memória de longo prazo como, por exemplo, as tarefas de fluência de aliteração utilizadas no presente estudo.

No que diz respeito à hipótese de Bowers (2001), de que a nomeação seriada rápida indexaria processos perceptuais responsáveis pelo mapeamento das características visuais de um estímulo, pode-se dizer que embora o presente estudo tenha refutado essa hipótese, ainda é muito cedo para descartá-la por completo, já que esse é o primeiro estudo que avaliou essa hipótese explicitamente por meio da técnica de análise fatorial confirmatória. Além disso, existem evidências de que a amplitude de atenção visual (do inglês, visual atention span) contribui de forma independente da consciência fonológica para a leitura em pessoas com dislexia (Bosse, Tainturier, \& Valdois, 2007). Nesse caso, existe a possibilidade de que a nomeação seriada rápida possa se relacionar, não com a velocidade de mapeamento visual, tal como propõe Bowers, mas sim com a amplitude de atenção visual (que não foi avaliada no presente estudo). 
Isso, inclusive, poderia ser uma explicação alternativa para os achados do estudo de Bowers et al. (1999). Dessa forma, é possível que a nomeação seriada rápida continue tendo uma relação com o processamento visual, porém uma relação diferente daquela proposta por Bowers. Assim sendo, seria interessante que estudos futuros pudessem investigar se existe uma relação entre a nomeação seriada rápida e a amplitude de atenção visual.

Os resultados do presente estudo levantaram duas evidências importantes sobre a relação entre a nomeação seriada rápida e a velocidade de processamento geral: (1) o modelo de mensuração no qual as tarefas de nomeação seriada rápida foram concebidas como medidas da velocidade de processamento geral não foi consistente com os dados, o que vai de encontro com a hipótese de Kail e Hall (1994) e ao encontro dos resultados relatados por Powell et al. (2007); e (2) os resultados da análise do modelo de regressão estrutural no qual a velocidade de processamento geral, a recuperação de códigos fonológicos da memória de longo prazo e o processamento visual foram concebidas como fontes de variações na nomeação seriada rápida indicaram que a velocidade de processamento geral foi a única variável que explicou, de forma significante, as variações na nomeação seriada rápida. Uma explicação possível para essas duas evidências é a de que a nomeação seriada rápida, de fato, não seja um mero subproduto da velocidade de processamento geral, mas por ser uma tarefa cognitivamente complexa que exige velocidade, integração e sincronicidade em diversos processos de natureza perceptual, mnêmica e motora (Wolf \& Bowers, 1999), acaba por sofrer os efeitos da velocidade de processamento, já que a última tem um efeito geral em diversos processos cognitivos e metalinguísticos (e.g., existem correlações significativas entre a inteligência geral e a consciência fonológica e morfológica - Anthony, Williams, McDonald, \& Francis, 2007; Kirby et al., 2012). O problema com essa hipótese alternativa é que, embora ela explique a relação entre a nomeação seriada rápida e a velocidade de processamento geral, ela é uma hipótese bastante difícil de operacionalizar e de investigar diretamente. Afinal, se a nomeação seriada rápida é o produto da orquestração sincrônica e eficiente de vários processos cognitivos de natureza diferente, quais seriam as relações relevantes a serem representadas entre esses processos e quais as medidas relevantes dos mesmos que deveriam ser incluídas em um modelo que visasse avaliar essa hipótese? Até o presente momento, a hipótese de Wolf e Bowers (1999) não parece ser explícita o suficiente de forma a propiciar esse tipo de teste.

\section{Considerações Finais}

Apesar dos resultados do presente estudo não oferecerem suporte para nenhuma das três hipóteses, eles trouxeram evidências de que, dentre as três variáveis pensadas como estando subjacentes à nomeação seriada rápida (processamento fonológico, velocidade de processamento geral e processamento visual), a velocidade de processamento geral foi a única que explicou as variações na nomeação seriada rápida de forma estatisticamente significante. Esse resultado ressalta a importância de que hipóteses mais específicas sobre como se daria a relação entre a nomeação seriada rápida, a velocidade de processamento e a leitura sejam desenvolvidas. Por exemplo, a relação entre a nomeação seriada rápida e a leitura estaria diretamente relacionada a um mero aumento na rapidez no processamento da informação que ocorre com a idade, conforme preconizado por Kail e Hall (1994), ou a relação entre a nomeação seriada rápida e a leitura seria mediada por outros fatores que, também, se relacionam com a velocidade de processamento geral?

Não há dúvidas de que a nomeação seriada rápida é uma tarefa cognitivamente complexa e que vários processos importantes devem funcionar adequadamente e serem coordenados para a sua realização. O que permanece sem resposta é: uma vez que parece haver uma relação entre essa variável e a velocidade de processamento geral, como o presente estudo evidenciou, de que forma específica se dá essa relação? Enquanto não conseguirmos obter essa resposta, a nomeação seriada rápida permanecerá sendo apenas uma boa ferramenta para rastreamento de crianças que têm ou poderão vir a ter dificuldade de leitura (considerando, é claro, a necessidade de se gerar normas para tal fim no Brasil). No entanto, infelizmente, a elaboração de programas eficazes de intervenção visando à prevenção e à remediação das dificuldades de leitura geradas por falhas no(s) processo(s) subjacente(s) à nomeação seriada rápida permanecerá difícil (de Jong \& Vrielink, 2004).

\section{Referências}

Anthony, J., Williams, J., McDonald, R., \& Francis, D. (2007). Phonological processing and emergent literacy in younger and older preschool children. Annals of Dyslexia, 57, 113-137.

Babayigit, S., \& Stainthorp, R. (2010). Component processes of early reading, spelling, and narrative writing skills in Turkish: A longitudinal study. Reading and Writing, 23, 539-568.

Barth, A., Catts, H., \& Anthony, J. (2009). The component skills underlying reading fluency in adolescent readers: a latent variable analysis. Reading and Writing, 22, 567-590.

Bishop, A., \& League, M. (2006). Identifying a multivariate screening model to predict reading difficulties at the onset of kindergarten: A longitudinal analysis. Learning Disability Quarterly, 29, 235-252.

Bosse, M., Tainturier, M., \& Valdois, S. (2007). Developmental dyslexia: The visual attention span deficit hypothesis. Cognition, 104, 198-230.

Bowers, P. (2001). Exploration of the basis for rapid naming's relationship to reading. In M. Wolf (Ed.), Dyslexia, fluency, and the brain (pp.41-63). Timonium, MD: York Press.

Bowers, P., Sunseth, K., \& Golden, J. (1999). The route between rapid naming and reading process. Scientific Studies of Reading, 3, 31-53.

Bowey, J., Mcguigan, M., \& Ruschena, A. (2005). On the association between serial naming speed for letters and digits and word-reading skill: Towards a developmental account. Journal of Research in Reading, 28, 400-422.

Bradley, L., \& Bryant, P. (1978). Difficulties in auditory organization as a possible cause of reading backwardness. Nature, 271, 746-747. 
Brady, S., \& Shankweiler, D. (1991). Phonological processes in literacy. Hillsdale, NJ: Lawrence Erlbaum.

Brizzolara, D., Chilosi, A., Cipriani, P., Filippo, G., Gasperini, F., Mazzotti, S., . . \& \& Zoccolotti, P. (2006). Do phonologic and rapid automatized naming deficits differentially affect dyslexic children with and without a history of language delay? A study of Italian dyslexic children. Cognitive Behavioral Neurology, 19, 141-149.

Cardoso-Martins, C., Haase, V., \& Wood, G. (1998). Bateria de Testes de Habilidades Fonológicas (Tradução e adaptação da Phonological Assessment Battery). Instrumento não publicado, Departamento de Psicologia, Universidade Federal de Minas Gerais, Belo Horizonte, Brasil.

Catts, H., Gillispie, M., Leonard, L., Kail, R., \& Miller, C. (2002). The role of speed of processing, rapid naming and phonological awareness in reading achievement. Journal of Learning Disabilities, 35, 509-524.

Cronin, V. (2011). RAN and double-deficit theory. Journal of Learning Disabilities, 46, 182-190.

Cutting, L., \& Denckla, M. (2001). The relationship of rapid serial naming and word reading in normally developing readers: An exploratory model. Reading and Writing: An Interdisciplinary Journal, 14, 673-705.

de Jong, P., \& van der Leij, A. (2003). Developmental changes in the manifestation of a phonological deficit in dyslexic children learning to read a regular orthography. Journal of Educational Psychology, 95, 22-40.

de Jong, P., \& Vrielink, L. (2004). Rapid automatic naming: Easy to measure, hard to improve (quickly). Annals of Dyslexia, $54,65-88$.

Denckla, M., \& Rudel, R. (1976). Rapid automatized naming (RAN): Dyslexia differentiated from other learning disabilities. Neuropsychology, 14, 471-479.

Di Filippo, G., Brizzolara, D., Chilosi, A., De Luca, M., Judica, A., Pecini, C., .. . \& Zoccolotti, P. (2006). Naming speed and visual search deficits in readers with disabilities: Evidence from an orthographically regular language (Italian). Developmental Neuropsychology, 30, 885-904.

Escribano, C. (2007). Evaluation of the double-deficit hypothesis subtype classification of readers in Spanish. Journal of Learning Disabilities, 40, 319-330.

Forster, L., \& Forster, C. (2003). DMDX: a windows display program with millisecond accuracy. Behavior Research Methods, Instruments and Computers, 35, 116-124.

Garson, D. (2012). Structural equation modeling. Asheboro, NC: Statistical Associates Publishers.

Georgiou, G., Parrila, R., Cui, Y., \& Papadopoulos, T. (2013). Why is rapid automatized naming related to reading? Journal of Experimental Child Psychology, 115, 218-225

Grigorenko, E., \& Naples, A. (2008). Single-word reading: Behavioral and biological perspectives. London: Lawrence Erlbaum Associates.

Justi, C., \& Roazzi, A. (2012). A contribuição de variáveis cognitivas para a leitura e a escrita no português brasileiro. Psicologia: Reflexão e Crítica, 25, 605-614.

Justi, C., Roazzi, A., \& Justi, F. (2014). São as tarefas de nomeação seriada rápida medidas do processamento fonológico? Psicologia: Reflexão e Crítica, 27, 1-11.

Kail, R., \& Hall, L. (1994). Processing speed, naming speed, and reading. Developmental Psychology, 30, 949-954.
Kail, R., Hall, L., \& Caskey, B. (1999). Processing speed, exposure to print, and naming speed. Applied Psycholinguistics, 20, 303-314.

Katzir, T., Kim, Y., Wolf, M., Morris, R., \& Lovett, M. (2008). The varieties of pathways to dysfluent reading comparing subtypes of children with dyslexia at letter, word, and connected text levels of reading. Journal of Learning Disabilities, 41, 47-66.

Kirby, J., Pfeiffer, S., \& Parrila, R. (2003). Naming speed and phonological awareness as predictors of reading development. Journal of Educational Psychology, 95, 453-464.

Kirby, J., Deacon, S., Bowers, P., Izenberg, L., Wade-Woolley, L., \& Parrila. R. (2012). Children's morphological awareness and reading ability. Reading \& Writing, 25, 389-410.

Kline, R. (2005). Principles and practice of structural equation modeling. London: The Guilford Press.

Landerl, K., \& Wimmer, H. (2008). Development of word reading fluency and spelling in a consistent orthography: An 8-year follow-up. Journal of Educational Psychology, 100, 150-161.

Lepola, J., Poskiparta, E., Laakkonen, E., \& Niemi, P. (2005). Development of and relationship between phonological and motivational processes and naming speed in predicting word recognition in grade 1. Scientific Studies of Reading, 9, 367-399.

Lervåg, A., Bråten, I., \& Hulme, C. (2009). The cognitive and linguistic foundations of early reading development: A Norwegian latent variable longitudinal study. Developmental Psychology, 45, 764-781.

Logan, J., \& Schatschneider, C. (2014). Component processes in reading: Shared and unique variance in serial and isolated naming speed. Reading and Writing, 27, 905-922.

Logan, J., Schatschneider, C., \& Wagner, R. (2011). Rapid serial naming and reading ability: The role of lexical access. Reading \& Writing, 24, 1-25.

López-Escribano, C., \& Katzir, T. (2008). Are phonological processes separate from the processes underlying naming speed in a shallow orthography? Journal of Research in Educational Psychology, 16, 641-666.

Meyer, M., Wood, F., Hart, L., \& Felton, R. (1998). Selective predictive value of rapid automatized naming in poor readers. Journal of Learning Disabilities, 31, 106-117.

Nelson, J., Lindstrom, J., Lindstrom, W., \& Denis, D. (2012). The structure of phonological processing, and its relationship to basic reading. Exceptionality: A Special Education Journal, 20, 179-196.

Plaza, M. (2003). The role of naming speed, phonological processing and morphological/syntactic skill in the reading and spelling performance of second-grade children. Special Issue on Language Disorders and Reading Acquisition, 1, 46-60.

Plaza, M., \& Cohen, H. (2004). Predictive influence of phonological processing, morphological/syntactic skill, and naming speed on spelling performance. Brain and Cognition, 55, 368-373.

Powell, D., Stainthorp, R., Stuart, M., Garwood, H., \& Quinlan, P. (2007). An experimental comparison between rival theories of rapid automatized naming performance and its relationship to reading. Journal of Experimental Child Psychology, 98, 46-68.

Puolakanaho, A., Ahonen, T., Aro, M., Eklund, K., Leppanen, P., Poikkeus, A., . . . \& Lyytinenl, H. (2007). Very early phonological and language skills: Estimating individual risk of reading disability. Journal of Child Psychology and Psychiatry, 48, 923-931. 
Schatschneider, C., Carlson, C., Francis, D., Foorman, B., \& Fletcher, J. (2004). Kindergarten prediction of reading skills: A longitudinal comparative analysis. Journal of Educational Psychology, 96, 265-282.

Seymour, P. (1986). Cognitive analysis of dyslexia. London: Routledge \& Kegan.

Torppa., Parrila, R., Niemi, P., Lerkkanen, M., Poikkeus, A., \& Nurmi, J. (2013). The double deficit hypothesis in the transparent Finnish orthography: A longitudinal study from kindergarten to Grade 2. Reading and Writing, 26, 1353-1380.

Verhagen, W., Aarnoutse, C., \& van Leeuwe, J. (2008). Phonological awareness and naming speed in the prediction of Dutch children's word recognition. Scientific Studies of Reading, 12, 301-324.

Wagner, R., \& Torgesen, J. (1987). The nature of phonological processing and its causal role in the acquisition of reading skills. Psychological Bulletin, 101, 192-212.
Wagner, R., Torgesen, J., Laughon, P., Simmons, K., \& Rashotte, C. (1993). Development of young readers' phonological processing abilities. Journal of Educational Psychology, 85, 83-103.

Wechsler, D. (2002). WISC-III - Escala de Inteligência Wechsler para Crianças: Manual (3a ed.). São Paulo: Casa do Psicólogo.

Wimmer, H., Mayringer, H., \& Landerl, K. (2000). The doubledeficit hypothesis and difficulties in learning to read a regular orthography. Journal of Educational Psychology, 92, 668-680.

Wolf, M., Bally, H., \& Morris, R. (1986). Automaticy, retrieval processes, and spelling: A longitudinal study in average and impaired readers. Child Development, 57, 988-1000.

Wolf, M., \& Bowers, P. (1999). The double-deficit hypothesis for the developmental dyslexias. Journal of Educational Psychology, 91, 415-438.
Recebido em 17.07.2013

Primeira decisão editorial em 10.04.2014

Versão final em 24.04.2014

Aceito em 24.04.2014 